\title{
SHOCK TEMPERATURE MEASUREMENTS IN
}

\author{
$\mathrm{Mg}_{2} \mathrm{SiO}_{4} \mathrm{AND} \mathrm{SiO}_{2}$ AT HIGH PRESSURES
}

Gregory A. Lyzenga* and Thomas J. Ahrens

\author{
Seismological Laboratory \\ California Institute of Technology
}

Pasadena, CA 91125

\begin{abstract}
Temperatures in the high pressure shock state have been determined by measurement of optical radiation from pure samples of forsterite $\left(\mathrm{Mg}_{2} \mathrm{SiO}_{4}\right), \alpha$-quartz, and fused silica. Shock waves of known amplitude were produced by tantalum flyer impact using a two-stage light gas gun. Shock pressures in the ranges $150-175 \mathrm{GPa}$ and $70-115 \mathrm{GPa}$ for $\mathrm{Mg}_{2} \mathrm{SiO}_{4}$ and $\mathrm{SiO}_{2}$ respectively were achieved, and temperatures in the range $4500-6800 \mathrm{~K}$ were measured. The observed temperatures in $\mathrm{Mg}_{2} \mathrm{SiO}_{4}$ are consistent with the occurrence of a shock-induced phase transition with a transition energy of $\sim 1.5 \mathrm{MJ} / \mathrm{kg}$. Measured Hugoniot temperatures versus pressure in both fused and crystalline $\mathrm{SiO}_{2}$ shocked to the stishovite regime suggest the occurrence of a previously unknown transition, beginning at pressures of approximately $107 \mathrm{GPa}$ and $70 \mathrm{GPa}$ for $\alpha-q u a r t z$ and fused quartz, respectively. The energies and temperatures appear to be consistent with the onset of melting of stishovite under shock loading.
\end{abstract}

\section{Introduction}

Compression of minerals by strong shock waves has been an important technique for the determination of equations of state of candidate earth materials. The major problem in the application of the resulting data has been in the evaluation of the thermal pressure component in the shock wave equation of state, and in uncertainty about the degree of thermodynamic equilibrium behind the shock front, particularly where shock-induced phase transitions are involved.

We have developed a high speed optical pyrometry technique to yield shock temperature measurements, and have applied it to light gas gun driven shock experiments [Lyzenga and Ahrens, 1979]. The two-stage gun operated by Lawrence Livermore Laboratory was used in this series of experiments, and LLL provided technical assistance in carrying out the experiments. The concepts employed are an outgrowth of the pioneering experiments carried out by Kormer et al [1965] on alkali halides. The resulting data provide important constraints on the thermal contribution to equations of state in minerals, as well as yielding critical data bearing on the question of the existence and energetics of shock-induced phase changes. The present pyrometry method requires optically uniform and transparent (between 450 and $800 \mathrm{~nm}$ ) samples, so that thermal radiation from the portion of the sample in a shock compressed state may pass through the unshocked sample to the detectors. The radiated energy is measured as a function of time in six narrow $(\sim 9 \mathrm{~nm})$ wavelength intervals across the visible spectrum, and recorded during the $200-400$ nsec $\left(2-4 \times 10^{-7} \mathrm{sec}\right)$ duration of shock propagation through the sample. Calibration of the pyrometer allows the observed levels to be converted to spectral radiance values (power radiated/solid angle/sample area/ spectral bandwidth). The observed spectral radiance versus time signals display a thermal equilibrium radiation spectrum, with an effective emissivity near unity. It is therefore believed that the temperatures

Table 1. $\mathrm{Mg}_{2} \mathrm{SiO}_{4}$ Shock Temperatures

$\begin{array}{ccl}\text { Pressure }(\mathrm{GPa}) & \text { Temperature }(\mathrm{K}) & \text { Emissivity } \\ 153 \pm 3 & 4490 \pm 100 & .66 \pm .08 \\ 166 \pm 3 & 4690 \pm 100 & .60 \pm .06^{\mathbf{1}} \\ 175 \pm 4 & 4950 \pm 100 & .53 \pm .03 \\ { }_{1} \text { interpolated value } & & \end{array}$

Copyright 1980 by the American Geophysical Union.
Table $2 \alpha-Q u a r t z$ Shock Temperatures

$\begin{array}{rccc}\text { Pressure (GPa) } & \text { Shock Direction } & \text { Temperature (K) } & \text { Emissivity } \\ 75.5 \pm .8 & 1(0001) & 4600 \pm 150 & .70 \pm .05 \\ 86.0 \pm 1.0 & \|(0001) & 4860 \pm 150 & .90 \pm .07 \\ 92.0 \pm 1.0 & 1(0001) & 5400 \pm 150 & .87 \pm .07 \\ 98.0 \pm 1.0 & 1(0001) & 5470 \pm 150 & .97 \pm .07 \\ 106.5 \pm 1.0 & \|(0001) & 5820 \pm 150 & .97 \pm .07 \\ 108.0 \pm 1.0 & 1(0001) & 5700 \pm 150 & .96 \pm .07 \\ 115.0 \pm 1.0 & \|(0001) & 4880 \pm 150 & .88 \pm .07\end{array}$

reported here are those of a well defined thermodynamic Hugoniot state. This observation is in agreement with the expectation that local thermal equilibrium should be achieved in a time short compared with the shock rise time.

Shock temperatures are reported for forsterite $\left(\alpha-\mathrm{Mg}_{2} \mathrm{SiO}_{4}\right)$, quartz $\left(\alpha-\mathrm{SiO}_{2}\right)$, and fused silica, in the regimes of what have been termed the high pressure phases. In the case of forsterite, this corresponds to what may be the dominant lower mantle mineral assemblage [Jackson and Ahrens, 1979]. Although the presumed high pressure silica phase, stishovite, may not be itself a constituent of the earth's interior [Ringwood, 1975], its properties derived from shock data [McQueen, et ah, 1963, Davies, 1972] have provided models of lower mantle silicate properties.

\section{Experimental Results}

The samples were mounted on $2 \mathrm{~mm}$ thick tantalum driver plates, which were impacted by tantalum flyer plates at speeds between 4.6 and $6.7 \mathrm{~km} / \mathrm{sec}$ using a two-stage light gas gun which propels $30 \mathrm{~mm}$ diameter projectiles. Since the tantalum equation of state is well known in the pressure range of interest, existing Hugoniot data for single crystal forsterite [Jackson and Ahrens, 1979], quartz, and fused silica [Wackerle, 1962] have been used to calculate shock pressure from measurements of impactor velocity, using the impedance match method [Al'tshuler, 1965].

The sample materials used were all synthetically prepared. Forsterite samples were cut from a single crystal boule grown by the Czochralski technique, and this material is the same as that used by Jacksan and Ahrens [1979]. Sample densities were measured by the Archimedean method to be $3.222 \mathrm{~g} / \mathrm{cm}^{3}$. Fused silica of stock commercial grade was supplied by Amersil Corp., and had a uniform initial density of 2.204 $\mathrm{g} / \mathrm{cm}^{3}$. Crystalline quartz supplied by Adolf Meller Co. had a density of $2.65 \mathrm{~g} / \mathrm{cm}^{3}$. All samples were lapped and polished to optical smoothness. High purity tantalum (density $16.66 \mathrm{~g} / \mathrm{cm}^{3}$ ) impactor and driver plates were supplied by Lawrence Livermore Laboratory.

In the experiments carried out on $\mathrm{Mg}_{2} \mathrm{SiO}_{4}$ (Table 1), the samples used were approximately $3 \mathrm{~mm}$ in thickness and the shock was propagated along the (001) direction (parallel to the $\mathrm{C}$-axis). The temperature uncertainties reflect the errors of measurement and in the least-squares fitting of the six spectral radiance values to mean values of emissivity and temperature. Implicit in the analysis is the assumption that the observed radiation is characterized by a thermal spectrum with emissivity independent of wavelength in the visible region.

In the case of $\alpha$-quartz, shots were fired with samples whose (0001) direction was oriented either perpendicular or parallel to the shock propagation direction (Table 2). These samples as well as those used in shots on fused silica (Table 3) were also approximately $3 \mathrm{~mm}$ in thickness. 
Table 3. Fused Quartz Shock Temperatures

$\begin{array}{rcl}\text { Pressure }(\mathrm{GPa}) & \text { Temperature }(\mathrm{K}) & \text { Emissivity } \\ 71.5 \pm 1.0 & 4700 \pm 150 & .79 \pm .05 \\ 80.0 \pm 0.5 & 5360 \pm 150 & .75 \pm .05 \\ 88.0 \pm 1.0 & 5300 \pm 300 & \sim 1.0^{1} \\ 90.0 \pm 1.5 & 5800 \pm 200 & .84 \pm .04 \\ 97.0 \pm 2.0 & 5650 \pm 200 & \sim 1.0^{1} \\ 102.5 \pm 2.0 & 6500 \pm 300 & .90 \pm 1.0 \\ 108.0 \pm 2.0 & 6800 \pm 300 & \sim 1.0\end{array}$

${ }^{1}$ lower quality data (not plotted in Figure 3 )

The pressure and temperature range of the data for forsterite (Figure 1), although relatively narrow, is in general agreement with the expected Hugoniot temperature on the basis of calculations [Ahrens et al 1969a]. In contrast, the shock temperatures observed in both forms of $\mathrm{SiO}_{2}$ display anomalous behavior. Notably, in the case of $\alpha$-quartz, an abrupt $1000 \mathrm{~K}$ decline in shock temperature occurs between pressures of 107 and $115 \mathrm{GPa}$ (Figure 2). Fused silica (Figure 3) displays a relatively smooth shock temperature curve which is more than $1000 \mathrm{~K}$ lower than expected values throughout the investigated region. Temperature points reported as of "lower quality" suffered from poor instrument calibration and were calculated under the assumption of a strict blackbody. Thus, the lower quality temperature points should be regarded as lower limit values. Further data are required to determine the pressure at which $\alpha$-quartz resumes a "normal" temperature increase, and whether at lower pressures, fused silica temperatures agree with the theoretical calculations discussed below.

\section{Interpretations}

Theoretical Hugoniot temperatures may be calculated for materials by assuming values for the Grüneisen parameter, $\gamma(\mathrm{V})=\mathrm{V}(\partial \mathrm{P} / \partial \mathrm{E})_{\mathrm{v}}$ and the specific heat. Consider the pressure $\mathbf{P}_{\mathbf{H}}$ along the Hugoniot of a material, and the pressure $P_{\mathrm{s}}$ at the same volume along the adiabat of isentropic compression from standard conditions. The Mie-Gruneisen assumption yields,

$$
P_{H}-P_{s}=(\gamma / V)\left(E_{H}-E_{s}\right) \text {. }
$$

$E_{H}$ and $E_{s}$ are the specific internal energies of the Hugoniot and isentropic states respectively, and $V$ is the specific volume. Here $E_{H}=1 / 2$ $P_{H}(V o-V)$ and $E_{s}=-\int P_{s} d V$. Therefore, knowledge of the Hugoniot pressure $\mathbf{P}_{\mathrm{H}}(\mathrm{V})$ and assuming a model for $\gamma(\mathrm{V})$ allows numerical solution for the unknown $\mathrm{P}_{s}(V)$ [Ahrens et al 1969b] Using this calculated isentrope and the known Hugoniot to determine the internal

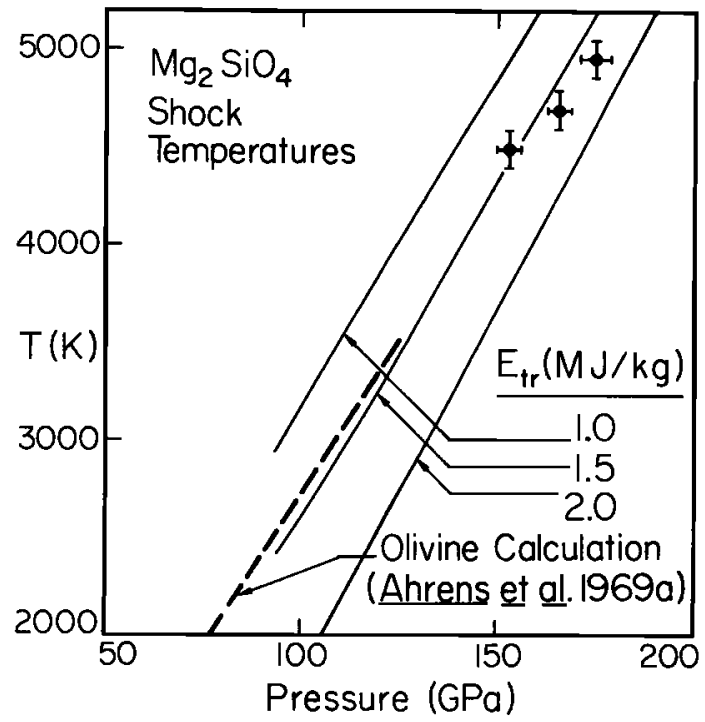

Figure 1. Measured and calculated shock temperatures versus pressure for forsterite. Calculations for three values of transition energy, $E_{t r}$ are shown.

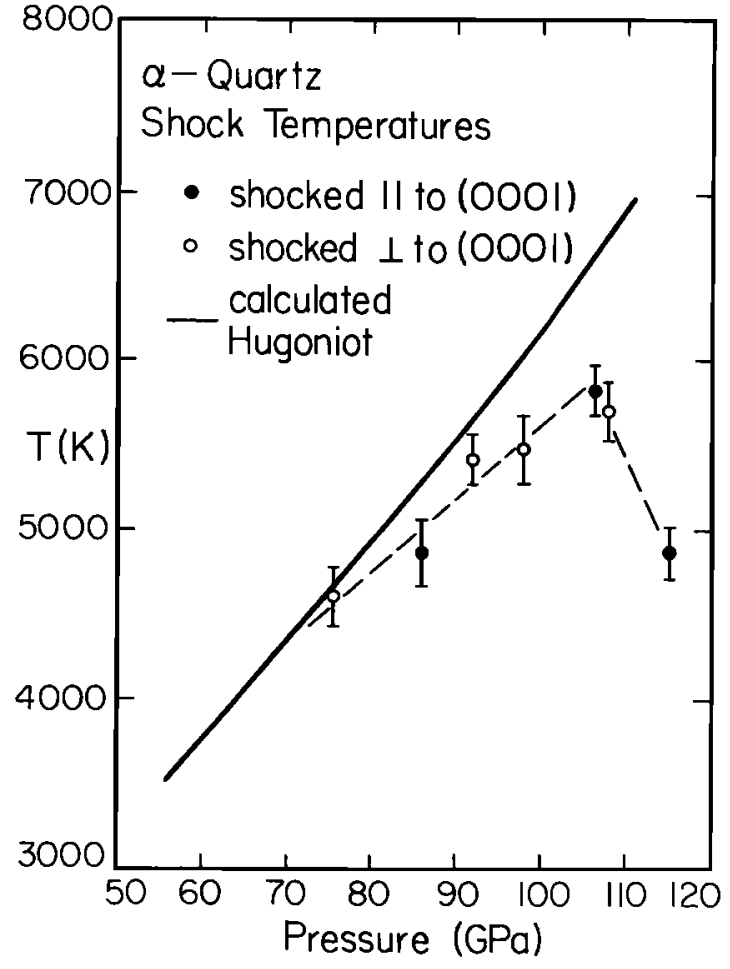

Figure 2. Shock temperatures versus pressure for single crystal $\alpha$-quartz.

energy difference $E_{H}-E_{s}$ through equation (1), the specific heat is used to calculate the temperature rise between the isentrope and Hugoniot at various values of $\mathrm{V}$.

In the case that shock compression incorporates transition to a high pressure phase (hpp), the analysis must take account of any change of enthalpy in the phase change. In the present work, this is accomplished by computing the isentrope starting from a "metastable Hugoniot" [McQueen et al 1967], or shock state locus centeréd on the putative high pressure phase. This metastable hpp Hugoniot is derived from the

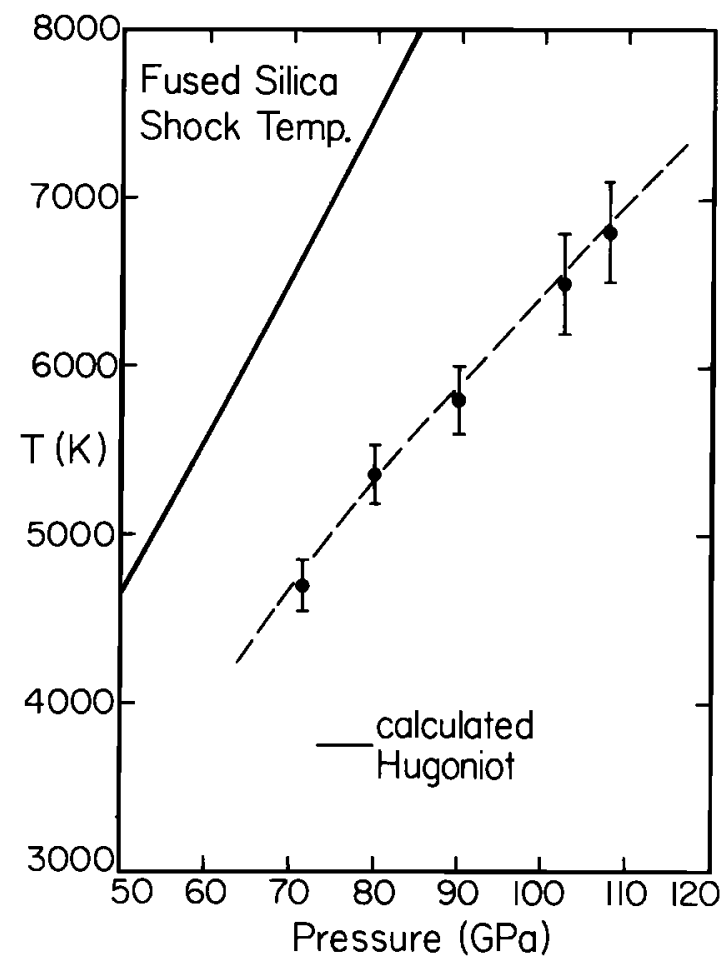

Figure 3. Shock temperatures versus pressure for fused silica. 


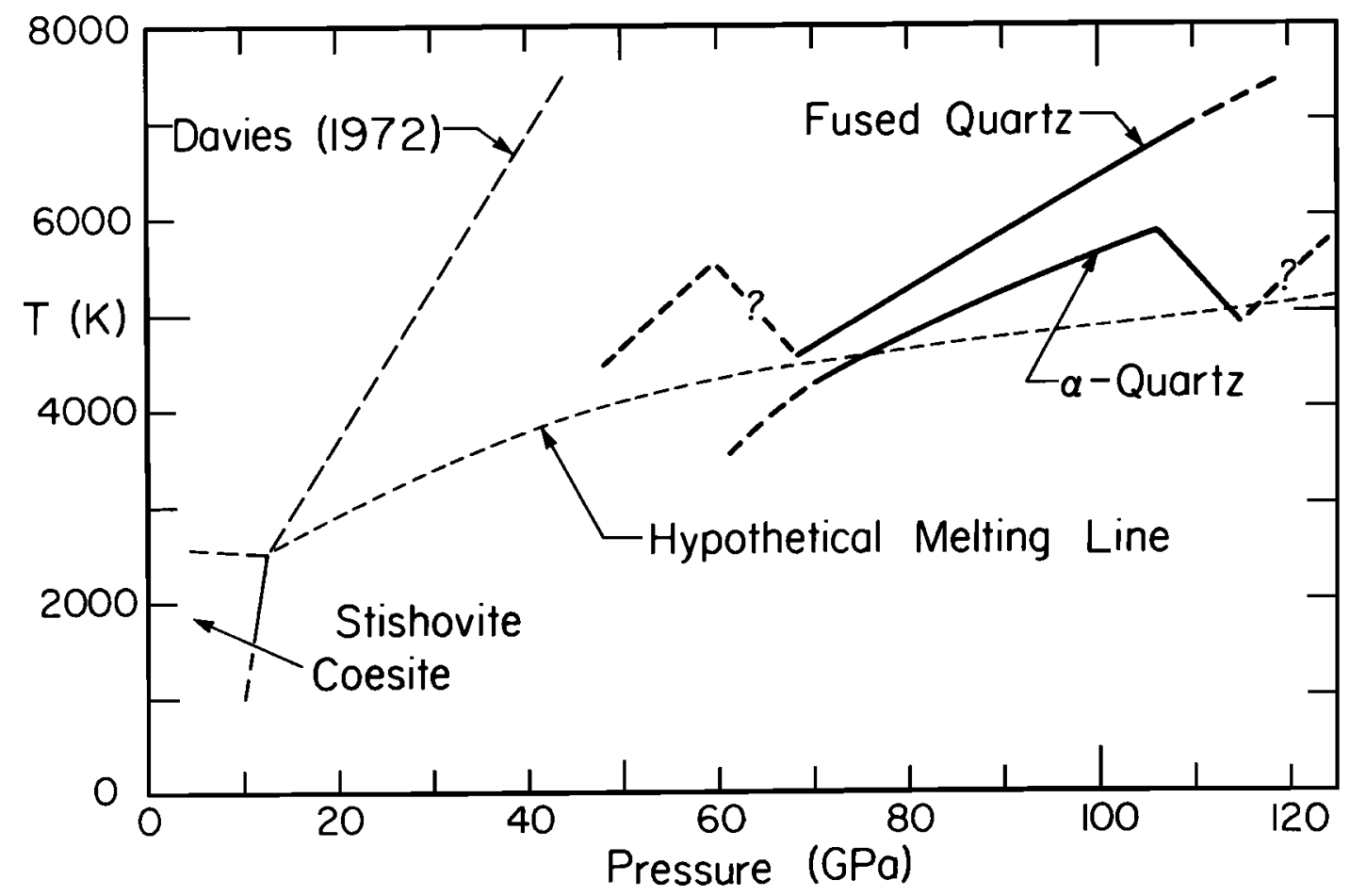

Figure 4. Hugoniot temperatures in the $\mathrm{SiO}_{2}$ phase diagram. Measured temperatures are shown with the estimated melting line of Davies [1972], and the hypothetical melting curve derived in this work. Melting line slope is $\sim 11 \mathrm{~K} / \mathrm{GPa}$.

principal experimental Hugoniot, given estimates of $E_{t r}$, the change in specific internal energy upon transition at standard conditions, and the Grüneisen parameter $\gamma(\mathrm{V}) . \mathrm{E}_{\mathrm{tr}}$ may be obtained from independent thermochemical data, or may be constrained by fitting values to the experimental temperature data.

If the particular metastable Hugoniot for which the initial high pressure phase has porosity so as to give it the same initial specific volume, Vo as the untransformed phase is chosen, then the metastable Hugoniot correction takes on a simple closed form. In that case, the amount $\Delta \mathrm{P}$ by which the metastable Hugoniot pressure exceeds the principal Hugoniot pressure in the hpp regime is,

$$
\Delta \mathrm{P}=\frac{\gamma}{\mathrm{V}} \mathrm{E}_{\mathrm{tr}}\left[1-\frac{\gamma}{2}\left(\frac{\mathrm{Vo}}{\mathrm{V}}-1\right)\right]^{-1}
$$

This expression is derived by applying the Mie-Grüneisen equation for the pressure offset due to differences in internal energy between the principal and metastable Hugoniots, and is valid only in the hpp regime of the principal shock locus. Given the computed isentrope $P_{s}(V)$, we assume the Debye model for specific heat to calculate Hugoniot temperatures from internal energy differences. For the high pressure phases considered, Debye temperatures of approximately $1000 \mathrm{~K}$ have been used.

Shock experiments on single crystal forsterite carried out by Jackson and Ahrens [1979] support the existence of shock-induced transformation to a high-pressure assemblage with a zero-pressure density of approximately $3.9 \mathrm{~g} / \mathrm{cm}^{3}$. Jackson and Ahrens [1979] could fit their data by assuming the olivine crystal structure changed to $\mathrm{MgSiO}_{3}$ (perovskite) and $\mathrm{MgO}$ (periclase) or alternatively, mixed oxides, $2 \mathrm{MgO}+$ $\mathrm{SiO}_{2}$. They made estimates of $\mathrm{E}_{\mathrm{tr}}=1.0 \pm 0.5 \mathrm{MJ} / \mathrm{kg}$ and $\gamma=(1.5 \pm 0.5)$ $\sigma^{1.5} \pm{ }^{.5}$ where $\sigma=\mathrm{V} / \mathrm{Vo}$ hpp is the compression relative to the hpp volume. Those values were used as starting models in fitting the forsterite data. The measured temperatures are consistent ' with theoretical calculations using $\mathrm{E}_{\mathrm{tr}}=1.5 \pm 0.3 \mathrm{MJ} / \mathrm{kg}$. While the data presently do not constrain $\gamma$, the above range of values are consistent with the measured temperatures, with the central value of the range $\gamma=1.5 \sigma^{1.5}$ giving the best agreement. Varying the volume dependence of $\gamma$ through the volume exponent weakly varies the pressure-temperature slope, but does not strongly change the fit to the data. There appears to be some problem in exactly fitting the observed temperature-pressure slope, but further data will be required to determine whether this discrepancy is real. The slope slope centered on a pressure of $170 \mathrm{GPa}$ is $(\mathrm{dT} / \mathrm{dP})_{H}=23 \mathrm{~K} / \mathrm{GPa} \pm 7$. In contrast, the range of values calculated by varying the volume exponent between zero and 2.0 is $34 \mathrm{~K} / \mathrm{GPa}+12$

In the case of $\mathrm{SiO}_{2}$, thermochemical data [Robie et al 1978] are available, assuming that the hpp is correctly identified as stishovite, the octahedrally coordinated $\mathrm{SiO}_{2}$ polymorph. The values of $\mathrm{E}_{\mathrm{tr}}$ for transition to stishovite from $\alpha$-quartz and fused silica used here are $0.822 \mathrm{MJ} / \mathrm{kg}$ and $0.675 \mathrm{MJ} / \mathrm{kg}$ respectively. Using silica shock compression data centered on quartz of different initial densities, $\gamma(V)$ has been estimated as $\gamma=0.950 \sigma^{(0.5)}$ using the results of Jeanloz and Richter [1979].

The calculated temperatures for $\alpha$-quartz show reasonable agreement with experiment (Figure 2) at lower pressures, but a significant discrepancy in temperature is seen above approximately $100 \mathrm{GPa}$. If the temperature drop is the manifestation of a shock-induced phase transition in a solid of classical 3R specific heat, it corresponds to a latent heat of $1.1 \mathrm{MJ} / \mathrm{kg}$. While the calculations succeed in predicting stishovite temperatures below the transition for states shocked from initial $\alpha$-quartz material, the fused quartz calculations fail to reproduce the experimental data. The data fall below the calculations with an offset of roughly the same magnitude as the temperature drop seen in shocked $\alpha$-quartz. We propose that a transition with $\sim 1000 \mathrm{~K}$ temperature decline also occurs on the fused silica Hugoniot, at a pressure just below the range investigated here. This conclusion is strengthened by the observations of McQueen, Fritz, and Hopson [1979], in which radiation from shocked $\mathrm{SiO}_{2}$ has been recorded photographically, and quantitatively analyzed using microdensitometry techniques. These observations indicate that just such a temperature decline occurs near $60-70 \mathrm{GPa}$ pressure, and that below this pressure, the observed temperatures agree more closely with the predictions. Further experiments using the present pyrometry technique may confirm this supposition in the near future. If this interpretation is correct, the stishovite shocked from initial fused silica displays a transition latent heat of roughly $1.2 \mathrm{MJ} / \mathrm{kg}$. These estimates of transition energy (the thermal expansion is taken to be small enough so that the latent heat is given by the temperature change divided by specific heat) are in the correct range for melting transitions. The large latent heat without an obvious large volume change speaks strongly for a 
melting transition as opposed to a solid state phase change. Experimental data and some theoretical suggestions [Stishov, 1975] support the observation that in the limit of high temperatures and pressure, the entropy of melting in a wide variety of substances is approximately $0.7-$ $0.9 \mathrm{R}$ per mole of atoms. In the present case of $\mathrm{SiO}_{2}$, this corresponds to an expected latent heat of $\sim 1.4-1.8 \mathrm{MJ} / \mathrm{kg}$, in agreement with the observed values. Further studies by Stishov [1975] suggest a high pressure limit to relative volume change on melting of $\Delta \mathrm{V} / \mathrm{V} \lesssim .03$ for some simple solids. If this holds for $\mathrm{SiO}_{2}$ we can use the Clausius-Clapeyron relation to estimate the slope of the melting line (Figure 4). This gives $(\mathrm{dT} / \mathrm{dP})_{\mathrm{m}} \lesssim 26 \mathrm{~K} / \mathrm{GPa}$ at a pressure of $90 \mathrm{GPa}$, in agreement with the trend of possible melting temperatures and pressures in $\alpha$-quartz and fused silica. The hypothetical phase line in Figure 4 for example, exhibits

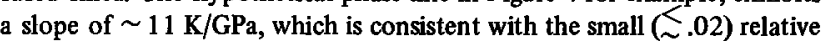
volume change required if the transition escaped notice in previous Hugoniot measurements. Furthermore, the Lindemann melting criterion [Grover, 1971] predicts a temperature-volume melting line slopé, given the value of the vibrational Grüneisen $\gamma$ of a Debye solid. The Lindemann prediction using the previously mentioned $\gamma(\mathrm{V})$ for stishovite is in good agreement with the observed V-T slope.

The proposed melting line shown in Figure 4 would indicate that for $\alpha-q u a r t z$ and fused silica, the Hugoniot curves penetrate considerably into the liquid field before the onset of melting and its attendant heat of fusion. Thus, the superheated solid is apparently metastable on the time scale of the shock front rise time, and the fusion is observed only after the equilibrium transition has been considerably "overdriven". While the data given here are consistent with melting of stishovite under shock, the inferred phase boundary disagrees strongly with that of Davies [1972].

In conclusion, the new $\mathrm{Mg}_{2} \mathrm{SiO}_{4}$ shock temperature data are in good agreement with previous calculations which assume a shock induced phase with a zero pressure density of $\sim 3.9 \mathrm{~g} / \mathrm{cm}^{3}$ and a heat of transformation $1.5 \pm 0.3 \mathrm{MJ} / \mathrm{kg}$. Further extension of the temperature measurements, especially toward lower values are planned, providing experimental difficulties with extraneous non-thermal radiation can be solved. In the case of $\mathrm{SiO}_{2}$, an apparent phase transition is observed in stishovite shocked from both the $\alpha$-quartz and fused silica states. This transition, which is accompanied by a large latent heat and small volume change may represent melting of the stishovite, in the manner of shock induced melting of $\mathrm{NaCl}$ and $\mathrm{KCl}$, [Kormer et al., 1965] or possibly the transformation to a yet denser polymorph, such as that having the fluorite structure. We note however that Liu [1978] inferred that such a transformation, if it occurs, does not take place at mantle pressures.

Acknowledgments. Supported under NSF Grant EAR 7812942 and the Department of Energy. The use of the Lawrence Livermore Laboratory light gas gun and electronics facilities are appreciated as is the help and encouragement of A. C. Mitchell, J. W. Shaner and W. J. Nellis. We appreciate the assistance of E. Gelle, J. Long and R. Smith of Caltech and D. Bakker, H. Martinez and W. C. Wallace of LLL. Many useful comments and suggestions on the manuscript and interpretations were provided by R. Jeanloz of Harvard University. Contribution No. 3332, Division of Geological and Planetary Sciences, California Institute of Technology, Pasadena, CA 91125.

*Supported by the Fannie and John Hertz Foundation

\section{References}

Ahrens, T.J., D.L. Anderson and A.E. Ringwood, Equations of state and crystal structures of high-pressure phases of shocked silicates and oxides, Rev. Geophys., 7, 667-707, 1969a.

Ahrens, T.J., C.F. Petersen and J.T. Rosenberg, Shock compression of feldspars, J. Geophys. Res., 74, 2727-2746, 1969 b.

Al'tshuler, L.V., Use of shock waves in high-pressure physics, Sov. Phys. Usp., 8, 52-91, 1965.

Davies, G.F., Equations of state and phase equilibria of stishovite and a coesitelike phase from shock-wave and other data, J. Geophys. Res., 77, 4920-4933, 1972.

Grover, R., Liquid Metal Equation of State Based on Scaling, J. Chem. Phys., 55, 3435-3441, 1971.

Jackson, I. and T.J. Ahrens, Shock-wave compression of single-crystal forsterite, J. Geophys. Res., 84, 3039-3048, 1979.

Jeanloz, R. and F.M. Richter, Convection, composition and thermal state of the lower mantle, J. Geophys. Res., 84, 5497-5504, 1979.

Kormer, S.B., M.V. Sinitsyn, G.A. Dirillov, and V.D. Urlin, Experimental determination of temperature in shock-compressed $\mathrm{NaCl}$ and $\mathrm{KCl}$ and of their melting curves at pressures up to $700 \mathrm{kbar}$, Sov. Phys. JETP, $21,689-700,1965$.

Liu, L., A fluorite isotype of $\mathrm{SnO}_{2}$ and a new modification of $\mathrm{TiO}_{2}$ : Implications for the earth's lower mantle, Science, 199, 422-425, 1978.

Lyzenga, G.A. and T.J. Ahrens, A multi-wavelength optical pyrometer for shock compression experiments, Rev. Sci Instr., 50, 1421-1424, 1979.

McQueen, R.G., J.N. Fritz, and J.W. Hopson, The temperature of Shocked $\mathrm{SiO}_{2}$ (abstract) EOS, 60, 952, 1979.

McQueen, R.G., J.N. Fritz, and S.P. Marsh, On the equation of state of stishovite, J. Geophys. Res, 68, 2319-2322, 1963.

McQueen, R.G., S.P. March and J.N. Fritz, Hugoniot equation of state of twelve rocks, J. Geophys. Res., 72, 4999-5036, 1967.

Rice, M.H., R.G. McQueen and J.M. Walsh, Compression of solids by strong shock waves, Solid State Physics, vol. 6, pp. 1-63, Academic Press, New York, 1958.

Ringwood, A.E., Composition and Petrology of the Earth's Mantle, p. 61 8, McGraw-Hill, New York, 1975.

Robie, R.A., B.S. Hemingway, and J.R. Fisher, Thermodynamic Properties of Minerals and Related Substances at $298.15 \mathrm{~K}$ and $1 \mathrm{Bar}$ $\left(10^{5}\right.$ Pascals) Pressure and at Higher Temperatures, pp. 216-221, Government Printing Office, Washington, D.C., 1978.

Stishov, S.M., The thermodynamics of melting of simple substances, Sov. Phys. Usp., 17, 625-643, 1975.

Wackerle, J., Shock-wave compression of quartz, J. Appl. Phys, 33, 922-937, 1962.
(Received October 9, 1979; accepted November 19, 1979.) 